\title{
Development of $\mathrm{CO}_{2}$ snow cleaning for in situ cleaning of $\mu \mathrm{CMM}$ stylus tips
}

\author{
Xiaobing Feng ${ }^{1}$, Peter K. Kinnell ${ }^{2}$, Simon Lawes ${ }^{1}$ \\ ${ }^{1}$ The University of Nottingham, Nottingham, United Kingdom \\ ${ }^{2}$ Loughborough University, Loughborough, United Kingdom \\ Email: s.lawes@,nottingham.ac.uk
}

\begin{abstract}
Contamination adhered to the surface of a $\mu \mathrm{CMM}$ stylus tip compromises the measurement accuracy of the $\mu \mathrm{CMM}$ system, potentially causing dimensional errors that are over ten times larger than the uncertainty of a modern $\mu \mathrm{CMM}$. In prior work by the authors, the use of a high pressure $\mathrm{CO}_{2}$ gas stream was demonstrated to achieve significant cleaning rate for a range of contaminant without damage to the stylus tip surface. This paper explores the practical challenges of achieving effective stylus tip cleaning in situ on $\mu \mathrm{CMM}$ systems. Two types of snow cleaning approaches were evaluated for their coverage of cleaning, thermal impact and gas flow forces. This work then presents a novel multi-nozzle prototype system using pulsed snow streams to achieve cleaning coverage over the entire stylus tip, and balances forces from the snow streams reducing drag force imparted by the gas stream to levels comparable to the probing force of $\mu \mathrm{CMMs}$, as well as allowing automated cleaning procedure integrated into a $\mu \mathrm{CMM}$ system.
\end{abstract}

Keywords: snow cleaning, $\mu \mathrm{CMM}$, precision cleaning, cleaning force.

\section{Introduction}

Contact metrology systems such as a micro-coordinate measuring machine ( $\mu \mathrm{CMM})$ are constantly pursuing smaller stylus size in order to keep up with the measurement demands for precisely manufactured components that are rapidly reducing in size. Typically, features with millimetre to micrometre size are expected to be measured with sub micrometre accuracy. The tip of a $\mu$ CMM stylus is usually smaller than $0.5 \mathrm{~mm}$ in diameter, and the smallest ruby tip stylus commercially available is $125 \mu \mathrm{m}$ in diameter, for use with Zeiss F25 $\mu$ CMM. The prototype $\mu$ CMM developed at the Swiss Federal Institute for Metrology METAS uses a stylus tip as small as $100 \mu \mathrm{m}$ [1]. Metallic tip spheres as small as $60 \mu \mathrm{m}$ have been fabricated in laboratories using electro-discharge machining and electrical chemical machining processes [2]. The 3D fibre probe developed by Werth uses a glass sphere down to $20 \mu \mathrm{m}$ in diameter. With both the stylus and measured features shrinking to the micrometre scale, the measurement error induced by debris has become increasingly significant and can no longer afford to 
be left unaddressed. During the probing operation contaminant particles are adhered onto the stylus tip surface and any subsequent measurements that coincide with this contact point will result in a dimensional error as indicated in Figure 1. A particle of a few micrometres in size could cause significant dimensional errors, which are orders of magnitude greater than those reported elsewhere [3, 4]. Despite best efforts, debris is often present on a brand new stylus and is unavoidably introduced to the stylus tip in various operations [5-7]. Figure 2 shows an example of such debris on a tip sphere made of ruby; the image was obtained using back-scattered electron (BSE) imaging in a scanning electron microscope (SEM).

The occurrence of the debris cannot be compensated through calibration of the stylus since any contact with the stylus during calibration will likely cause debris to attach, detach, move or deform, hence negating the calibrated results. Therefore, regular cleaning of the stylus tip is essential in maintaining measurement accuracy.

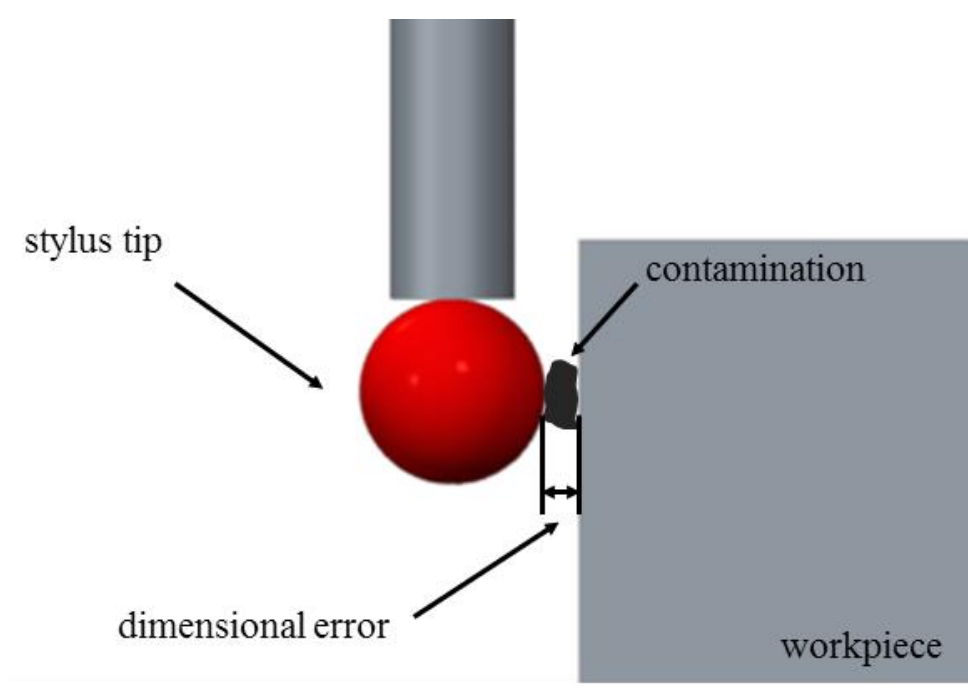

Figure 1 Illustration of the dimensional error of a $\mu \mathrm{CMM}$ caused by surface contamination

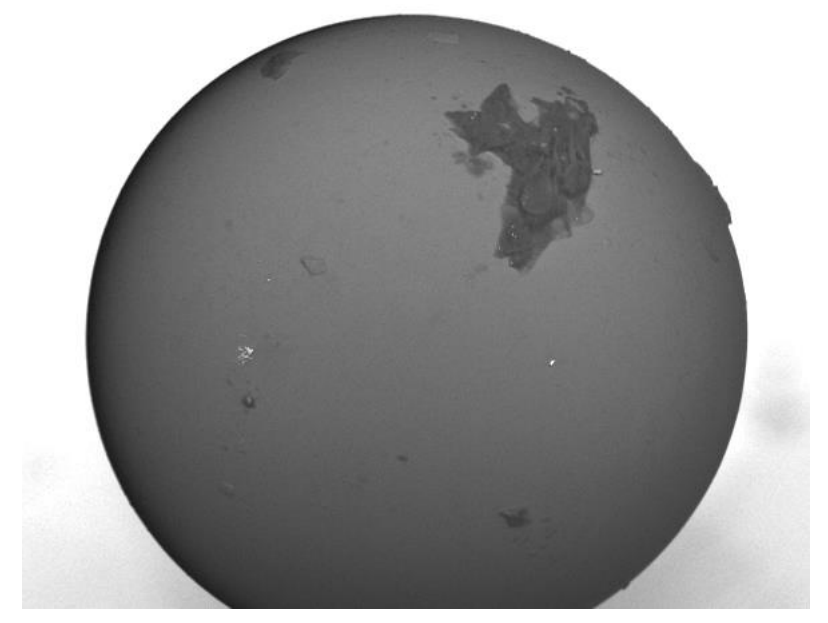

Figure 2 BSE SEM image of debris found on a new unused stylus of $0.3 \mathrm{~mm}$ tip diameter 
Debris-induced error is not quantified in current standards, with ISO 10360-5:2010 requiring only the cleaning of stylus tips before any qualification procedure in adherence to procedures given in the manufacturer's operating manual [8]. At present no standard for the cleaning of $\mu \mathrm{CMM}$ styli exists. A survey conducted by the authors among eight research entities within Europe, including national metrology institutes, university research centres and industrial instrument makers, has indicated that cleaning procedures of CMM styli vary significantly. While larger stylus tips with over $0.5 \mathrm{~mm}$ diameter are often cleaned with a cloth, an ultrasonic bath, solvents or a combination of the above, cleaning is rarely attempted on smaller styli. Previous studies $[9,10]$ have investigated cleaning of $\mu$ CMM styli with ultrasonic bath, plasma cleaner, snow cleaning and using precision energy beams. Focused ion beams were used [9] to target specific debris and particle removal proved effective. However, this method required knowledge of the location of debris, and local thermal damage was found on the stylus tip after cleaning. Where electron beams [9] and laser beams [10] were applied in a wide area irrigation fashion, particle removal was not as effective and global thermal damage was observed on the tip surface. In contrast, snow cleaning was found to be more effective at cleaning a range of contamination than ultrasonic bath and plasma cleaning while leaving no surface damage to the stylus tip. However, the shaft of the stylus may be damaged when the force imparted on the stylus by the high pressure stream of $\mathrm{CO}_{2}$ and $\mathrm{N}_{2}$ becomes too high. As such, optimised stream conditions for lower drag forces imparted by the gas stream are important in order to minimise the risk of stylus damage; a direct measurement of the drag force during cleaning is required to demonstrate that the method is safe for in situ $\mu \mathrm{CMM}$ cleaning.

A disadvantage of all currently available cleaning procedures for $\mu \mathrm{CMM}$ styli is that they require unmounting of the stylus from the CMM probe; this is not ideal as it disturbs the measurement procedure, involves human intervention and risks damage to the stylus and results in the need for recalibration of the stylus sphericity. An in situ cleaning procedure where remounting of the stylus is not required is highly desired, but is also difficult to achieve mainly due to coverage and risk of damage to the stylus. This study aims to address these specific challenges and explores in situ cleaning of $\mu \mathrm{CMM}$ stylus tips using $\mathrm{CO}_{2}$ snow. The paper starts by defining a methodology in section 2 to evaluate the two most common types of snow cleaning approaches: pure $\mathrm{CO}_{2}$ snow cleaning and assisted snow cleaning. Section 3 investigates potential damage to styli from snow streams, cleaning efficacy in terms of coverage and thermal effects. Based on the findings of the evaluation, a prototype device for in situ snow cleaning of $\mu \mathrm{CMM}$ styli has been developed in section 4; and the benefit of such a stylus cleaning system is demonstrated. Finally, section 5 concludes the contributions of this study.

\section{Methodologies of evaluation}

Previous studies by the authors have demonstrated the capability of the snow cleaning method to remove a range of contaminant without causing damage to the stylus tip surface, this study will expand that 
investigation to consider the practical considerations of applying this method to in situ $\mu \mathrm{CMM}$ stylus cleaning namely the coverage of the cleaning effect, the force exerted by the gas flow and potential damage to the stylus from thermal shock. Two prominent snow cleaning approaches currently available were evaluated. As there are no standards or previously described evaluation studies of this kind, it was first necessary to define these.

\subsection{Snow cleaning and assisted snow cleaning}

The snow cleaning process removes contamination by spraying solid $\mathrm{CO}_{2}$ particles onto a surface. The expansion of liquid $\mathrm{CO}_{2}$ propelled through an orifice facilitates the formation of gas and fine solid $\mathrm{CO}_{2}$ particles. Solid $\mathrm{CO}_{2}$ particles in the jet stream exit the nozzle at high speed and dislodge surface contamination upon impact. The high momentum in solid particles makes the cleaning efficacy significantly higher than an equivalent velocity compressed air jet. The particles also evaporate quickly and leave no trace behind [9], making it a dry cleaning process and abrasion free compared to grit blasting approaches.

Two types of snow cleaning approaches are commonly used: pure $\mathrm{CO}_{2}$ snow cleaning and assisted snow cleaning. A pure $\mathrm{CO}_{2}$ snow cleaning device typically consists of (1) $\mathrm{CO}_{2}$ cylinder, (2) hose, (3) a nozzle with an orifice and particular geometry and (4) a trigger mechanism often in the shape of a handgun, as shown in Figure 3(a). The assisted snow cleaning device utilises additional compressed gas (typically air or nitrogen) to assist the acceleration of $\mathrm{CO}_{2}$ particles and boost cleaning power. Most assisted snow cleaning devices are more complex as they require an additional cylinder, a coaxial delivery route and an electronic controller, as shown in Figure 3(b).

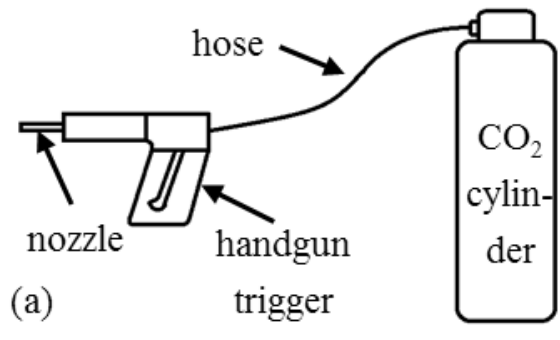

Figure 3 Illustration of typical (a) pure $\mathrm{CO}_{2}$ and (b) assisted snow cleaning devices

Dimensions of the internal geometry of the nozzles used in the two snow cleaning units are shown in Figure 4. Liquid $\mathrm{CO}_{2}$ passes through the orifice of the nozzle used in the pure $\mathrm{CO}_{2}$ device. After entering an expanded space, a mixture of solid particles and gas is formed, as shown in Figure 4(a). With the assisted device, snow particles and assisting gas are transported coaxially through the nozzle and mixed at the exit, as illustrated in Figure 4(b). 


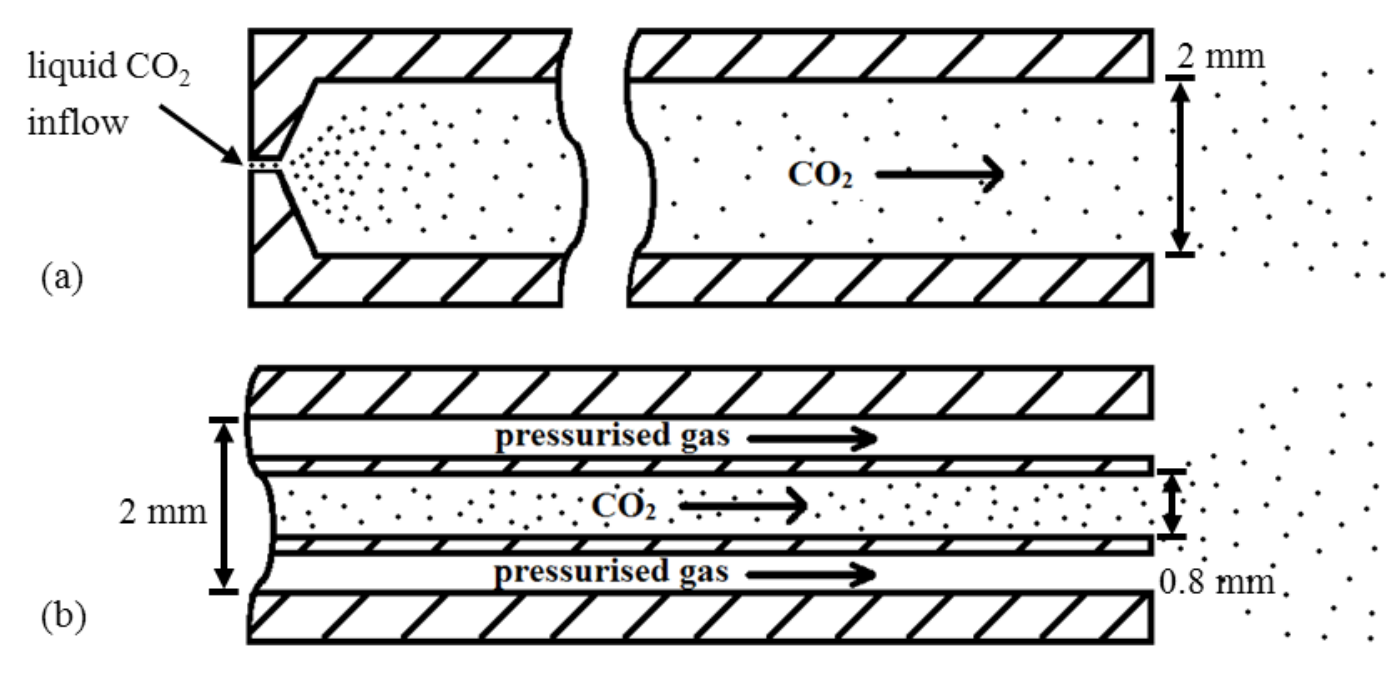

Figure 4 Illustration of nozzle internal geometries used for two evaluated snow cleaning approaches using (a) pure $\mathrm{CO}_{2}$ stream and (b) assisted snow stream

The main advantage of assisted snow cleaning is improved cleaning power due to higher particle velocity. In addition, the mixed stream induces less thermal impact than a pure $\mathrm{CO}_{2}$ stream due to higher temperature of the mixed stream. The electrical controller offers useful functions such as switching of stream source and adjustable flow conditions without modification to the mechanical setup. In comparison, a pure $\mathrm{CO}_{2}$ snow cleaning device is smaller and simpler in design, allowing more freedom for integration with the CMM system.

This study investigated both types of snow cleaning approaches: a handgun unit that used only $\mathrm{CO}_{2}$ and an assisted unit using additional nitrogen gas, as shown in Figure 5. The handgun unit (K1-05, Applied Surface Technologies) operated with a manual handgun trigger. The standoff distance between the stylus and the nozzle was adjustable, while stream pressure and flow condition can be adjusted by varying $\mathrm{CO}_{2}$ supply pressure and nozzle geometry. No electronic controller was required for the handgun unit. The assisted unit (SNOPEN, Cleanlogix) used additional nitrogen gas to accelerate the snow particles. The standoff distance was adjusted manually while thrust pressure of the assisting gas was adjustable through an electronic controller. Cleaning was triggered by foot pedals with two available modes: (1) with $\mathrm{CO}_{2}$ assisted by $\mathrm{N}_{2}$ and (2) with only $\mathrm{N}_{2}$ gas. Due to the difference in device makeup and power range, the two snow cleaning units were evaluated not at typical settings, but with comparable drag force of a few millinewtons. Details of the settings used and drag forces are discussed further in section 3 . 

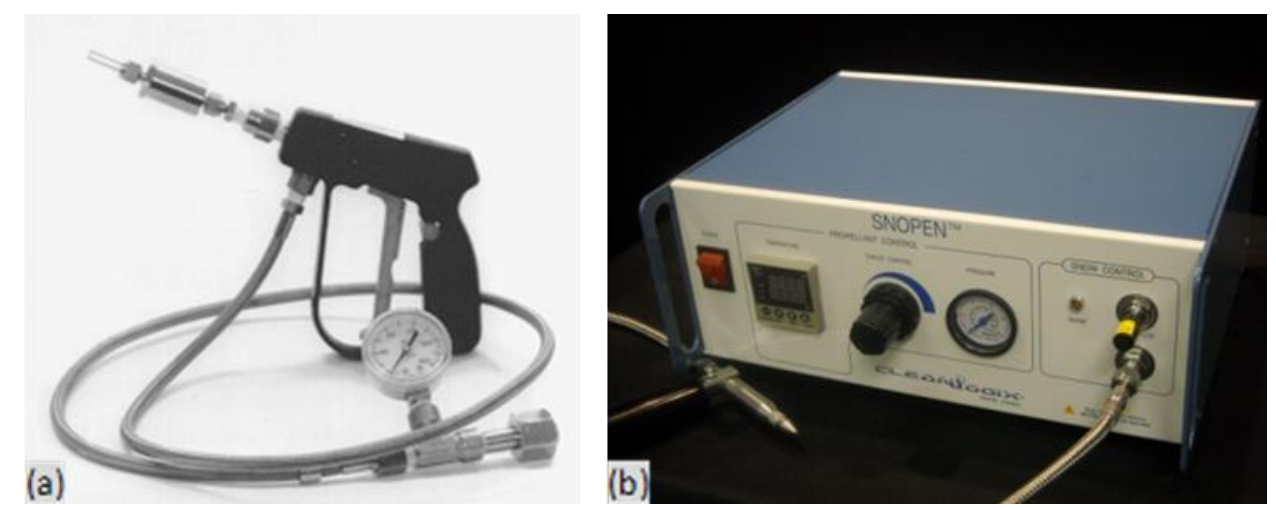

Figure 5 Two snow cleaning devices: (a) pure $\mathrm{CO}_{2}$ unit (courtesy, Applied Surface Technologies) and (b) assisted unit (courtesy, Cleanlogix)

\subsection{Assessing risk of damage}

The fragile nature of the $\mu \mathrm{CMM}$ stylus requires the cleaning process to be gentle. For utmost precision, a $\mu \mathrm{CMM}$ system is equipped with styli of very small dimension and highly sensitive probes operating with very low contact force, usually at a few millinewtons or less. For the commercially state-of-theart Zeiss F25 $\mu \mathrm{CMM}$ where the smallest stylus tip of $125 \mu \mathrm{m}$ diameter is used, the specified typical probing force is $0.5 \mathrm{mN}$ [9]. The $\mu \mathrm{CMM}$ probe developed at METAS also operates at probing forces below $0.5 \mathrm{mN}$ with stylus tip of $100 \mu \mathrm{m}$ diameter [1]. The fibre probe developed by Werth also achieves sub millinewton probing force. As the probing force is indicative of the physical limits of the styli, this study considered a cleaning technique to be safe if the cleaning force is within the same order of the probing force of a $\mu \mathrm{CMM}$, which ranges from less than half millinewton to a few millinewtons $[1,5,9]$. The risk of mechanical damage to the stylus increases with the amount of drag force imparted on the stylus during cleaning by the gas stream. As such, this study has measured the drag force during snow cleaning approaches and compared them with typical probing force range of $\mu \mathrm{CMMs}$. In the absence of precision force sensors, a mechanical/optical setup as depicted in Figure 6 was developed to measure the imparted drag force in addition to holding the stylus. 

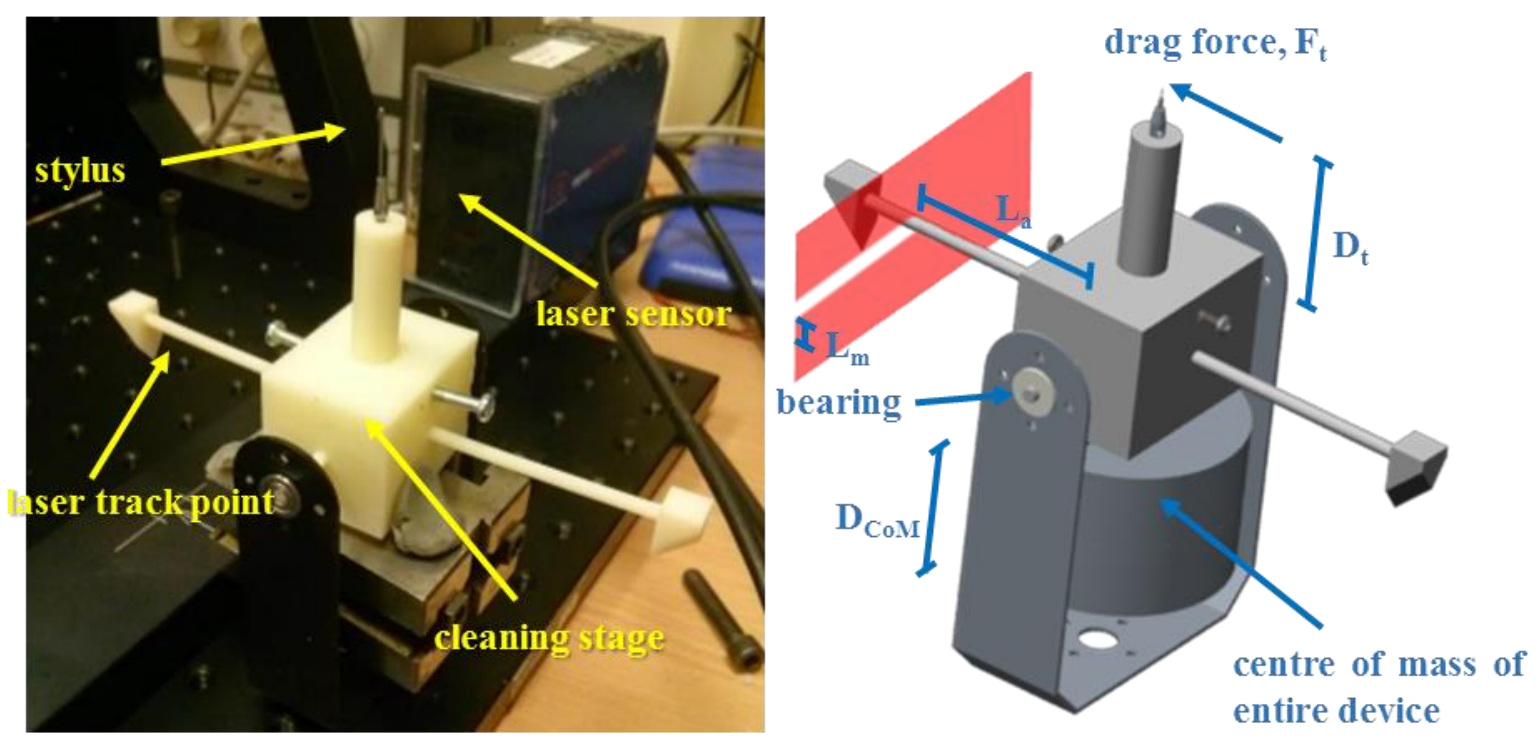

Figure 6 Hardware setup for measuring drag force during cleaning [11]

The 3D printed main body is supported by a U-shape bracket through a pair of bearings. The body holds the stylus and is loaded with a weight underneath. During cleaning, the imparted drag force induces a torque that causes the body to rotate about the pivot point by a small angle, which is determined by measuring the linear displacement of the extended arm with a laser micrometer (optoCONTROL 2600, MICRO-EPSILON). Using the principle of moment equilibrium, the drag force on the stylus can be calculated using Eq. 2-1.

$$
\mathrm{F}_{\mathrm{t}}=\operatorname{mg} \frac{D_{C o M}}{D_{t}} \frac{\mathrm{L}_{m}}{L_{a}}
$$

where $F_{t}$ is the drag force on the stylus tip, $m$ is the combined mass of the main body, additional weight and the stylus, $g$ is the gravitational constant, $D_{t}$ is the distance between the stylus tip and the rotational axis, $D_{C o M}$ is the distance between the centre of gravity of the device (main body, weight and stylus) and the rotational axis, $L_{m}$ is the linear displacement measured by the micrometer and $L_{a}$ is the distance from the measurement position on the extended arm to the rotational axis.

The laser micrometre has a specified repeatability of $\pm 1 \mu \mathrm{m}$ and linear accuracy of $\pm 3 \mu \mathrm{m}$ over $40 \mathrm{~mm}$ measurement range, converting into a repeatability of $\pm 0.027 \mathrm{mN}$ and linear accuracy of $\pm 0.08 \mathrm{mN}$ in force measurement. In order to determine the true force measurement error of the mechanical/optical setup, a comparison between applied known force and measured force has been carried out. The known force was applied by placing known weights on the other extended arm not being measured by the laser. The weights were measured using a precision analytical balance (PW254, Adam Equipment), which provided an equivalent repeatability of $0.002 \mathrm{mN}$ in applied force. The comparison, as shown in Figure 7, indicates a maximum error of $0.025 \mathrm{mN}$ within $1 \mathrm{mN}$ range and $0.091 \mathrm{mN}$ within $5 \mathrm{mN}$ range. Therefore, this mechanical/optical setup provides adequate accuracy within the scope of this study. 
In addition, potential abrasion to the stylus tip surface resulting from impact with snow particles was examined using an SEM. Thermal damage from contact with the snow stream was also investigated.

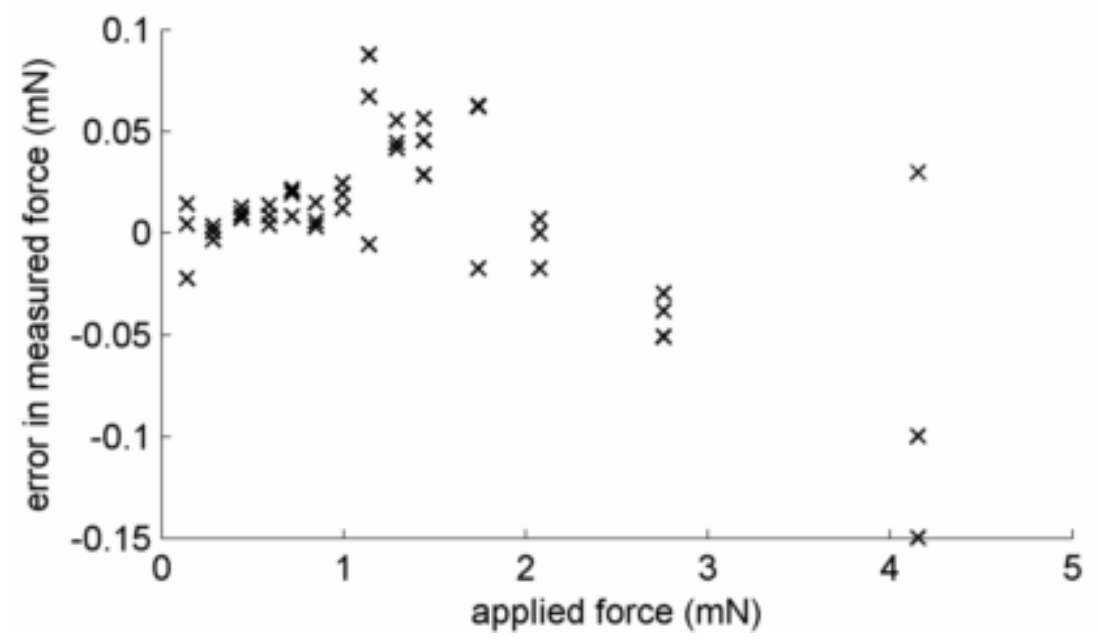

Figure 7 Force measurement error of the mechanical/optical setup

\subsection{Evaluating cleaning efficacy}

To evaluate any cleaning process, it is useful if there is an ability to create well defined artificial contamination. To define an artificial contamination strategy it is necessary to first understand the typical contamination characteristics of styli. Common sources of contamination on a $\mu \mathrm{CMM}$ stylus include [9]:

1. Organic residue left on the sample, such as traces of lubricant used during manufacturing, skin residue due to handling and dust in the air;

2. Metallic debris left on the sample, such as chips formed during cutting, ejected debris during electro-discharge machining and loose particles during additive manufacturing or lithography;

3. Debris detached from the sample surface during measurement, where microscale 'sharp' features on the sample surface are broken due to the extremely high local contact stress;

4. Debris on the dirty master sphere that has not been regularly cleaned;

5. Dust from the air that settled onto the stylus after prolonged exposure;

6. And chemical reaction between the stylus and the sample.

$\# 1, \# 2$ and \#5 are the primary sources of contamination. \#1 and \#2 can be minimised through proper cleaning of the sample (e.g. in an ultrasonic bath), but cannot be completely eliminated. \#3 is more likely to happen when measuring rough surfaces with sharp peaks. \#4 and \#6 can be addressed by cleaning the master sphere before calibration and avoiding chemical affinity between stylus tip material and the sample. Organic residue is typically soft and prone to deform during contact, which limits the amount of dimensional error it induces; however, this also makes it easily spread across the surface, leading to further cross contamination. Metallic debris is less prone to deform, and therefore induces larger dimensional error than organic debris with similar size. Prolonged contact of debris with the 
stylus tip can lead to strong chemical bonds which can make it impossible to remove the debris. Such contamination conditions should be avoided through regular cleaning. In general, an effective cleaning technique needs to be able to remove dust, organic and metallic debris from the stylus tip without exerting sufficient force to damage the fragile stylus.

Based on the above analysis, two approaches have been selected to prepare the contaminated styli for subsequent cleaning experiments: (1) by repeated probing of metrological samples and (2) by aerosol spray coating of micro particles.

The repeated probing approach produced contamination as would be found in normal measurement operations. For that purpose, the samples used in the study had been previously cleaned in an ultrasonic bath after being manufactured. In order to produce controlled contamination level, surface roughness comparator plates (Microsurf series, Rubert \& Co.) were used to contaminate the styli after undergoing the following preparation procedure:

1. cleaned with isopropanol in an ultrasonic bath for $5 \mathrm{~min}$ to remove existing contamination;

2. immersed in used cutting fluid (ROCOL V-cut EP) for $5 \mathrm{~min}$;

3. left to dry in the air for $5 \mathrm{~min}$, and

4. cleaned again with isopropanol in an ultrasonic bath for $5 \mathrm{~min}$.

Figure 8 shows a stylus tip being contaminated after probing on a prepared comparator plate for 300 contacts.

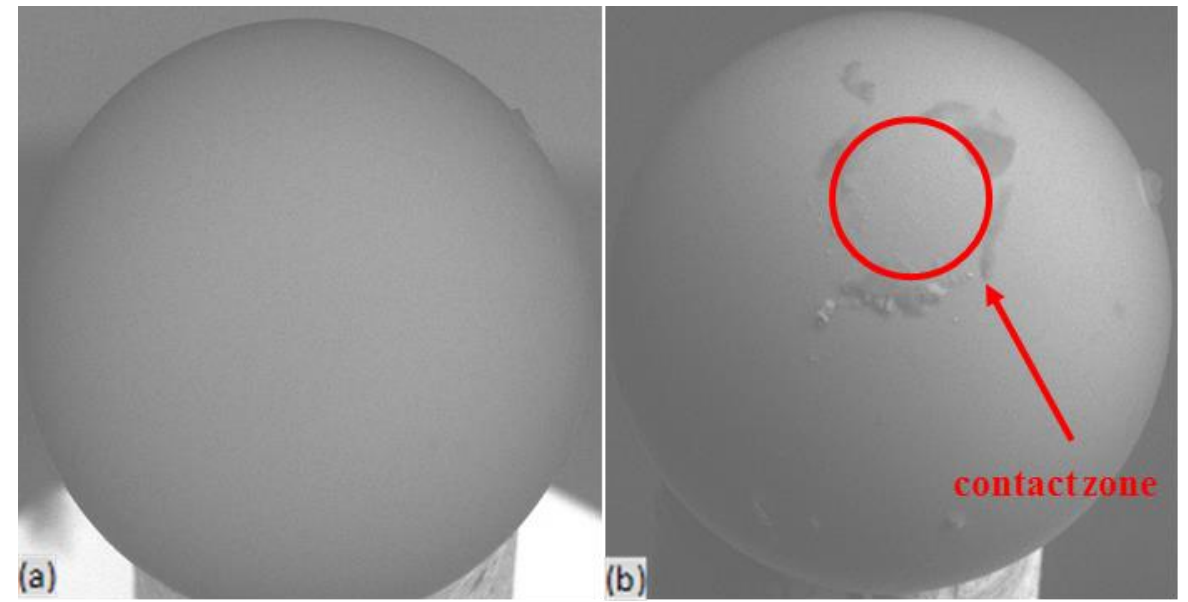

Figure 8 SEM image of a $0.3 \mathrm{~mm}$ stylus tip (a) before and (b) after 300 probing contacts

While the stylus was contaminated with debris picked up from the sample, the central area of the stylus, which is the point at which contact was made with the sample surface, remained relatively clean, suggesting the repeated probing action and contact force has resulted in debris in the central area being squeezed out of the contact zone. Various types of contamination was found on contaminated styli, which was used to evaluate cleaning efficacy of $\mathrm{CO}_{2}$ snow cleaning.

Due to the stochastic distribution of debris on the sample surface, contamination on the prepared stylus cannot be precisely replicated for each test. For repeated cleaning evaluations, a second approached was 
adopted to prepare the contaminated stylus by spraying the stylus tip with a developer spray typically used for non-destructive testing evaluations (SKD-S2, Spotcheck). The developer spray consists of particles of calcium carbonate and talc that are dissolved in alcohol; the alcohol quickly evaporated upon spraying and produced a relatively uniform white coating on the target surface, in this case around the stylus tip. A stylus with $1 \mathrm{~mm}$ tip diameter contaminated with this method is shown in Figure 9. The different shades of colour in Figure 9(a) is due to the reflection of the stylus shaft beneath it; the bright ring is due to reflection of the illumination source of the microscope.

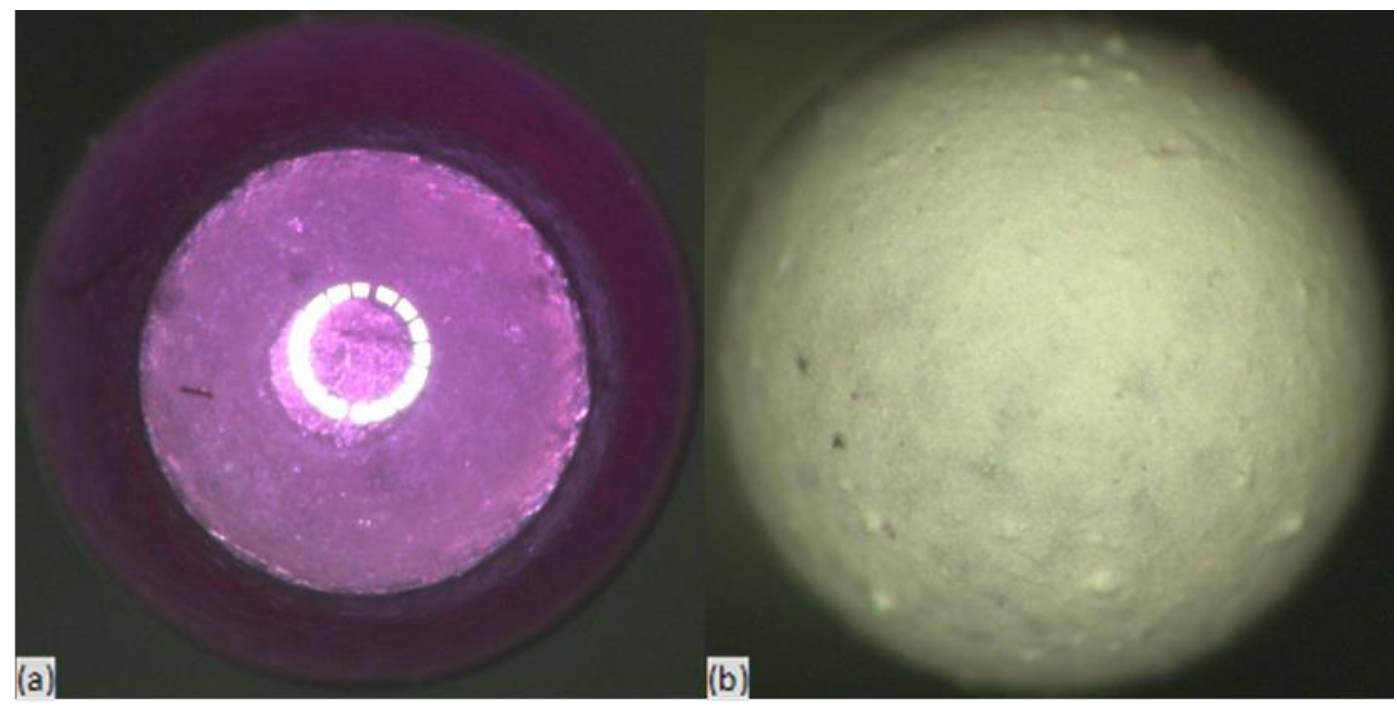

Figure 9 Microscope image of a CMM stylus with $1 \mathrm{~mm}$ tip diameter (a) before and (b) after being coated with developer particles

The degree of contamination produced with this approach can be replicated reasonably well, and the uniform coating of particles was used to examine cleaning coverage, where occluded areas not exposed to the snow stream would remain coated.

\section{Evaluation of snow cleaning}

When considering cleaning of microscale components such as a $\mu \mathrm{CMM}$ stylus, the fragile nature and small size of the stylus are of particular concern. As a result, this investigation focused on the drag force caused by the snow stream, cleaning efficacy in terms of coverage and thermal effects.

\subsection{Drag force}

External force is exerted onto the stylus upon impact with the snow stream. Figure 10 shows a stylus tip with $1 \mathrm{~mm}$ tip diameter, contaminated with developer particles, being exposed to a snow stream approaching at a direction normal to the stylus shaft. While the front hemisphere of the stylus tip in contact with the snow stream is being cleaned, contaminant particles on the back hemisphere remain 
intact. Due to the low temperature of the stream, snow particles have begun to accumulate on the front hemisphere as seen in the figure.

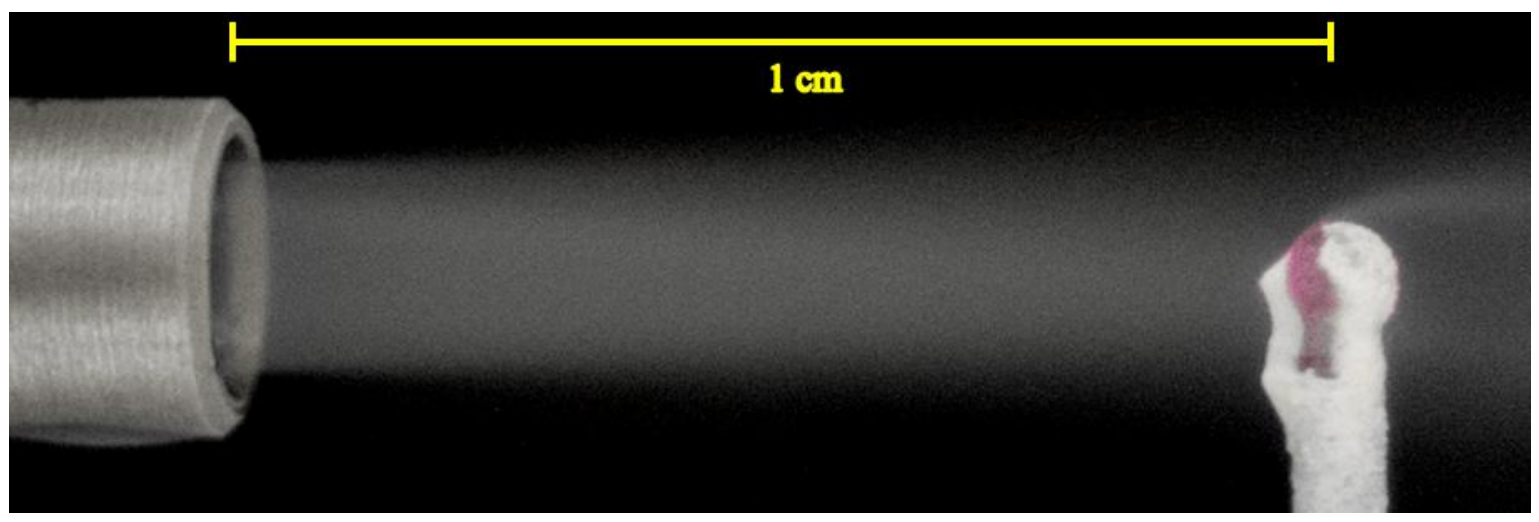

Figure 10 A stylus with $1 \mathrm{~mm}$ tip diameter being cleaned by a $\mathrm{CO}_{2}$ snow stream at a standoff distance of $1 \mathrm{~cm}$, illustrating the well-controlled non-divergent flow

Since the primary cleaning mechanism is momentum transfer, cleaning efficacy increases with faster particle velocity, which also leads to higher drag force. Therefore, evaluation of the cleaning efficacy is only useful when compared in a similar drag force range.

In order to identify the range of device settings for the two units to operate at comparable drag force levels, the two units were first operated within their typical settings recommended by the manufacturer for cleaning of small objects. Figure 11 shows the drag force values measured within a long period of over $30 \mathrm{~s}$, where the handgun unit was operated at $5 \mathrm{~mm}$ standoff distance and the assisted unit at $5 \mathrm{~cm}$ standoff distance and thrust pressure of 3 bar.

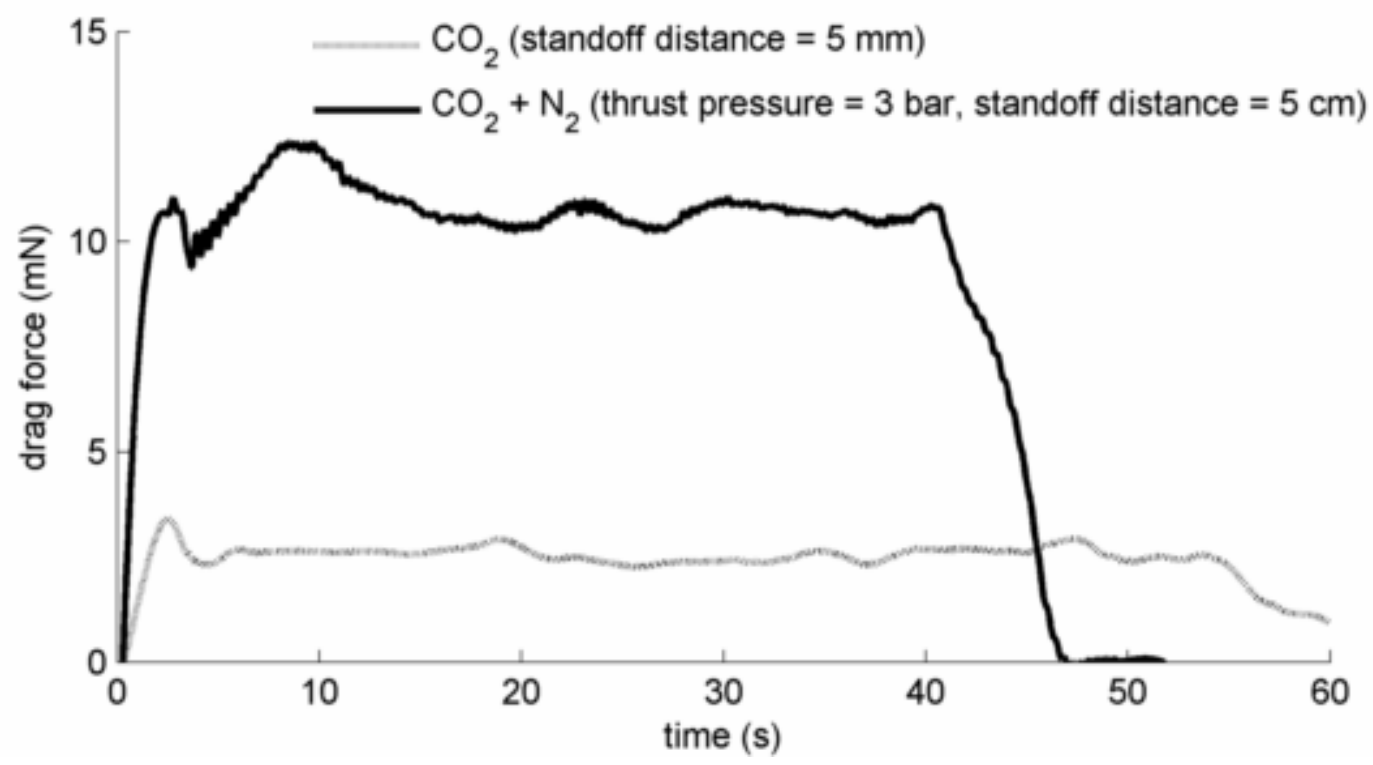

Figure 11 Graph of imparted drag force from gas stream developing over a $30 \mathrm{~s}$ test, demonstrating the dramatic difference in force exerted on the stylus by the two approaches 
Both curves recorded the start and end of the cleaning processes. The snow stream was switched off after approximately $41 \mathrm{~s}$ and $55 \mathrm{~s}$ respectively for the assisted unit and the handgun unit. After the streams were triggered $(t=0 \mathrm{~s})$, drag force values appeared to overshoot before reaching relatively stable levels. The overshoot in the curves was mainly due to the inertia of the rotating body, since the imparted drag force was calculated using measured rotation. As such, only the stable phase after overshoot was considered to reflect true imparted drag force values.

After the streams were switched off, the residual $\mathrm{CO}_{2}$ left in the nozzle and the hose caused the stream pressure to dissipate gradually over a few seconds. The rate of dissipation depended on the volume of residual gas, which was why the stream used in the assisted unit dissipated much faster than that of the handgun unit.

The assisted unit induced significantly higher drag force despite being placed 10 times farther from the stylus. This was due to the internal setup of the device, especially the addition of nitrogen gas as suggested in Figure 12, where the force imparted onto the stylus was measured when only assisting $\mathrm{N}_{2}$ gas was applied over a range of standoff distance and thrust pressure values. Error bar shows standard deviation of 5 repeated tests.

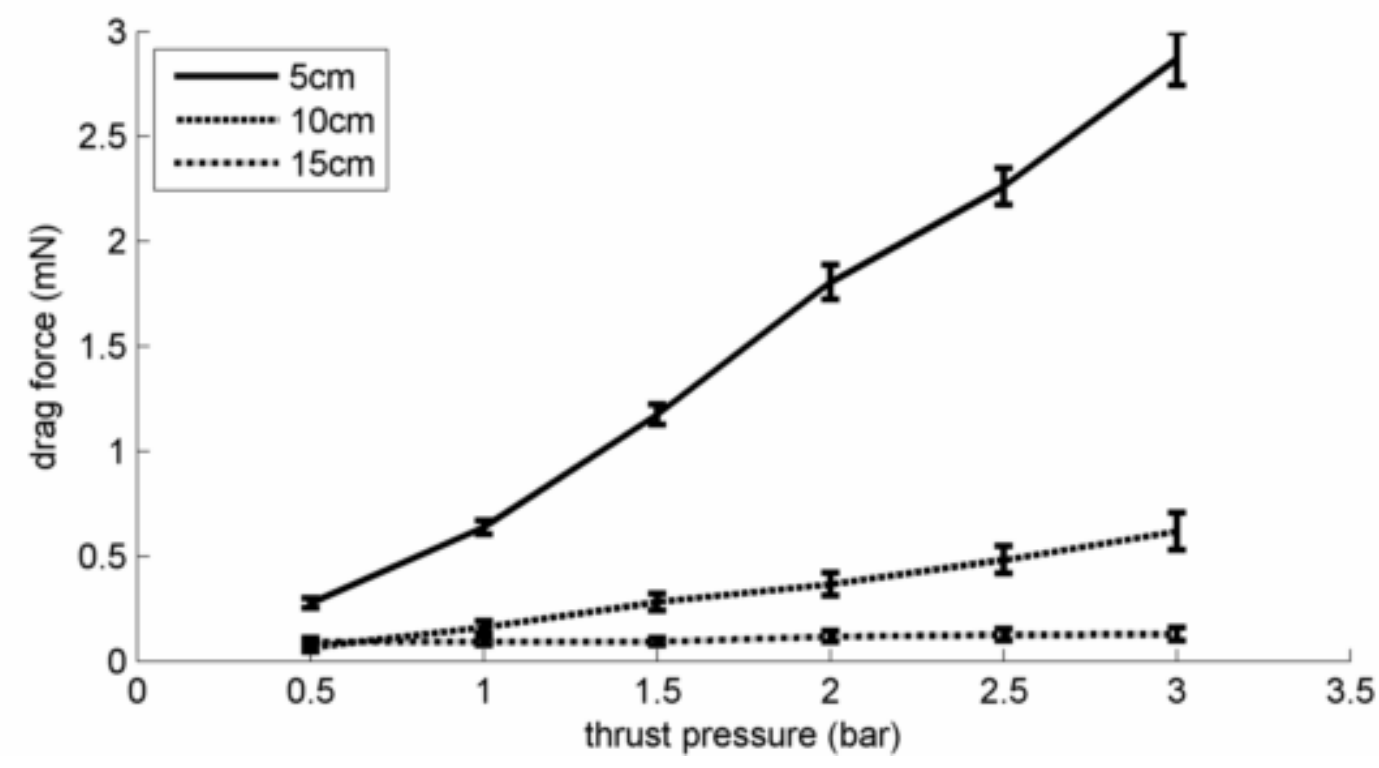

Figure 12 Imparted drag force on the stylus using assisting $\mathrm{N}_{2}$ gas only. Error bars show one standard deviation.

Imparted drag force was found to increase with closer standoff distance and higher thrust pressure. At $15 \mathrm{~cm}$ standoff distance, the drag force diminished below measurable threshold, indicating that the pressure of the $\mathrm{N}_{2}$ gas had completely dissipated beyond $15 \mathrm{~cm}$ distance. However, at $5 \mathrm{~cm}$ standoff distance and thrust pressure of 3 bar the drag force due to assisting $\mathrm{N}_{2}$ only, which contributed to approximately $25 \%$ of the total drag force as shown in Figure 11, had already exceeded that of the handgun unit at a much shorter standoff distance of $5 \mathrm{~mm}$. This suggested that the assisted unit was 
required to operate at longer standoff distance and low thrust pressure in order to keep the imparted drag force comparable with the handgun unit. The corresponding range of settings was subsequently determined and listed in Table 1, for each cleaning unit to operate at a drag force below $3 \mathrm{mN}$, which was considered by the authors as acceptable for $\mu \mathrm{CMM}$ styli.

Table 1 Range of settings involved in the evaluation

\begin{tabular}{|l|c|c|}
\hline & handgun unit & assisted unit \\
\hline standoff distance $(\mathrm{cm})$ & $0.5,1,2,3,4,5$ & 15 \\
\hline thrust pressure (bar) & N/A & $0.5,1,1.5,2,2.5,3$ \\
\hline
\end{tabular}

Figure 13 shows the force imparted onto a stylus with $1 \mathrm{~mm}$ tip diameter by each cleaning unit with the above listed settings. Error bar shows standard deviation of 10 repeated tests, where measurement under unstable flow conditions is excluded.
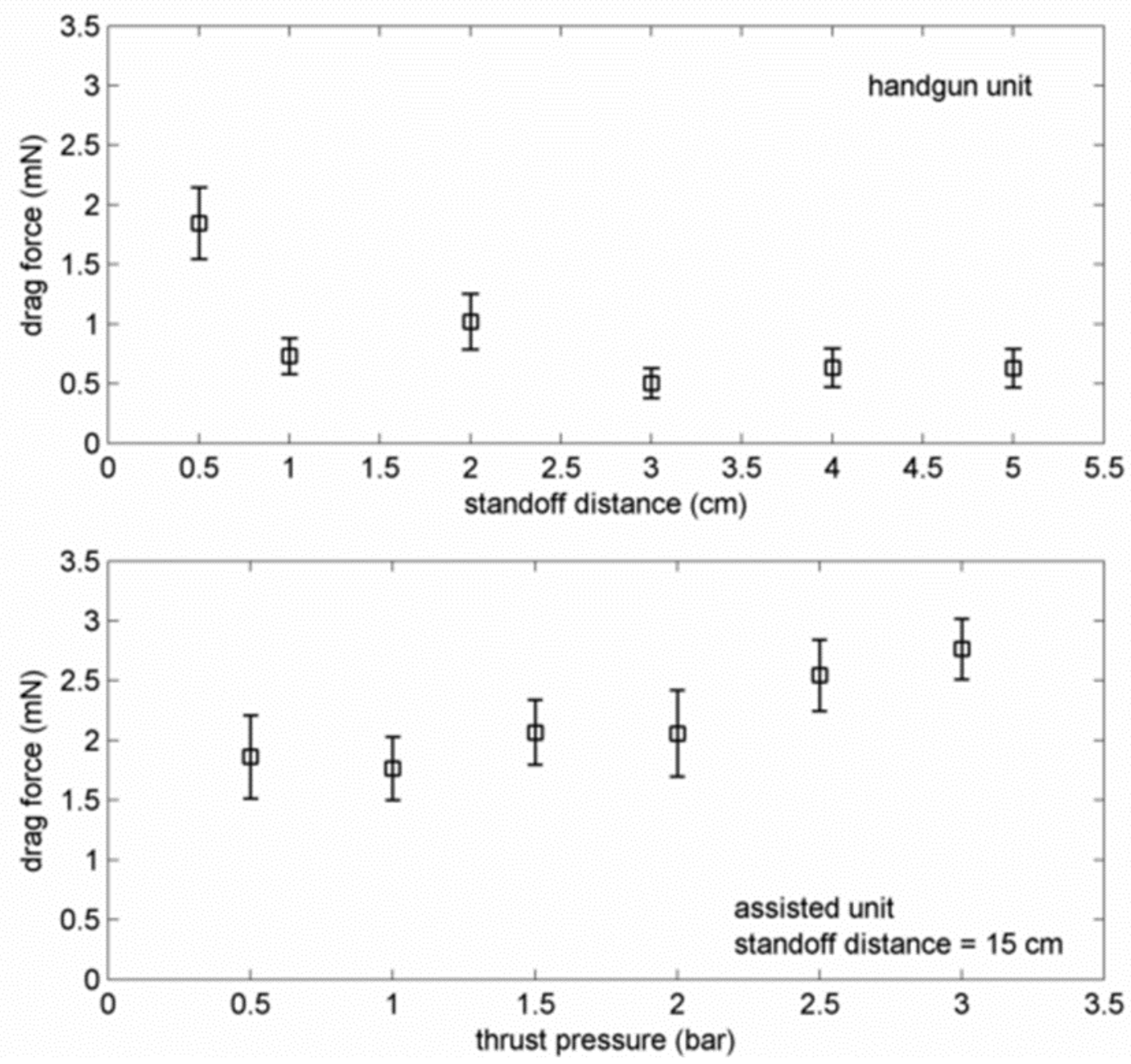

Figure 13 Imparted drag force during snow cleaning using the handgun unit (top) and the assisted unit (bottom). Error bars show one standard deviation 
The force imparted by the handgun unit was found to decrease with standoff distance, with diminished decreasing rate beyond $1 \mathrm{~cm}$. The force imparted by the assisted unit was found to increase with the thrust pressure of the assisting gas. Based on the previous indication that the assisting gas with thrust pressure below 3 bar would completely dissipate at $15 \mathrm{~cm}$ distance, the stream would reasonably also be considered as a pure $\mathrm{CO}_{2}$ upon impact with the stylus. In this case, the assisting gas contributed to the drag force indirectly by accelerating the snow particles, as indicated by elevated drag force with increasing thrust pressure. This agreed with the reasoning that drag force increases with flow rate and particle velocity.

Both units had successfully produced drag force values below $3 \mathrm{mN}$. Moreover, the handgun unit using a pure $\mathrm{CO}_{2}$ stream was able to achieve a drag force close to the probing force of the Zeiss F25, the METAS $\mu \mathrm{CMM}$ stylus and Werth 3D fibre probe at a much shorter standoff distance.

\subsection{Cleaning efficacy}

Cleaning tests have been conducted using both units operating at millinewton and sub-millinewton imparted drag force levels shown in Figure 13. Due to the difficulties in accurately reproducing the initial contamination conditions on the styli, especially with repeated probing approach, this study focused on qualitative evaluation of cleaning efficacy with an emphasis on cleaning coverage.

A stylus of $1 \mathrm{~mm}$ tip diameter, previously contaminated with developer particles, was snow cleaned using the handgun unit at $1 \mathrm{~cm}$ standoff distance, as shown in Figure 14.

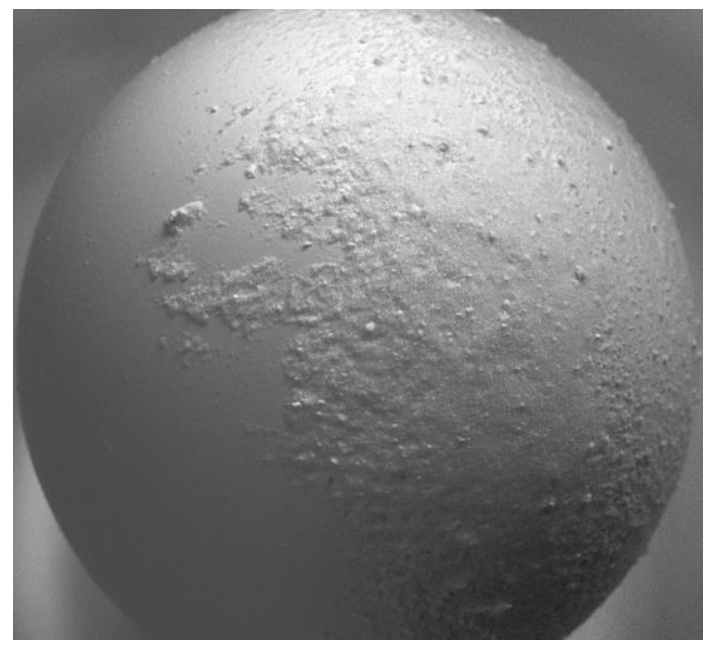

Figure 14 BSE SEM image of a CMM stylus with $1 \mathrm{~mm}$ tip diameter after cleaning with a $\mathrm{CO}_{2}$ snow stream for less than a second

After less than one second of exposure to the snow stream, the left hemisphere surface was significantly cleaned of debris. The other hemisphere on the occluded side was unexposed to the snow stream and therefore cleaning was not achieved. This was true for both types of snow cleaning approaches, which presented a challenge for the in situ cleaning application, as most $\mu \mathrm{CMM}$ systems do not allow rotation 
of the stylus around the shaft axis. This challenge will be addressed by the developed prototype, which is discussed in section 4 .

Figure 15 shows a stylus with $1 \mathrm{~mm}$ tip diameter contaminated through repeated probing and after $30 \mathrm{~s}$ of assisted snow cleaning at $15 \mathrm{~cm}$ standoff distance while rotating the stylus gradually for full coverage.

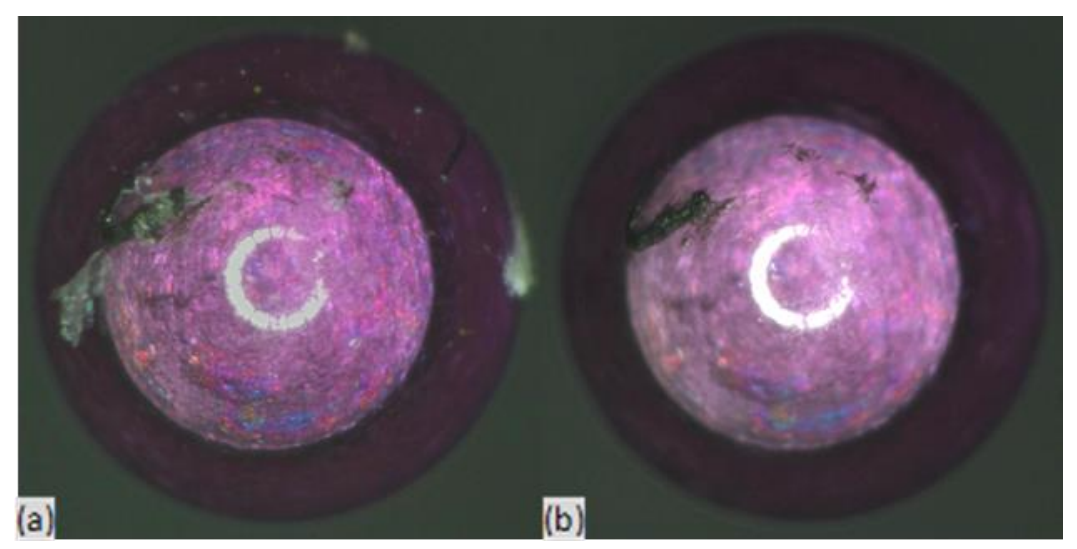

Figure 15 Microscope images of a CMM stylus with $1 \mathrm{~mm}$ tip diameter (a) contaminated with metallic particles and (b) after snow cleaning while rotating the stylus

Metallic debris, fibre, dust and organic compounds were found on the stylus tip after repeated probing. Debris size ranged from a few micrometres to over several hundred micrometres. After $30 \mathrm{~s}$ of snow cleaning with the assisted unit, the majority of debris was successfully removed including the large metallic debris, fibre and smaller particles. However, some debris remained which appeared to be better adhered to the tip surface.

The cleaning tests have suggested that debris does respond to higher cleaning forces and extended exposure to the snow stream. This agrees with the findings in a previous study [10], where subsequent cleaning of the stylus shown in Figure 15 was attempted by brushing a cotton swab and a soft feather against the stylus tip. No further cleaning was achieved until over 10 times higher drag force was used, upon which the remaining debris was only partly removed. This further indicated that snow cleaning efficacy was proportional to the force of the gas stream and that higher cleaning capability could potentially be achieved if a stronger stream can be used. In addition, no visible surface damage (e.g. abrasion) was found on the stylus tip under SEM. The use of coaxial compressed gas boosted the cleaning performance of the assisted unit. However, the settings used in the study were near the lowest power available, where lower drag force cannot be achieved. As a result, it was considered less suitable than the handgun unit for cleaning $\mu \mathrm{CMM}$ styli.

\subsection{Thermal effects}

Although extended cleaning time has been found to improve cleaning efficacy, caution needs to be taken during prolonged exposure. As the stylus temperature decreases over time, snow particles are 
more likely to build up on the stylus surface in forms determined by the flow condition, as shown in Figure 16.

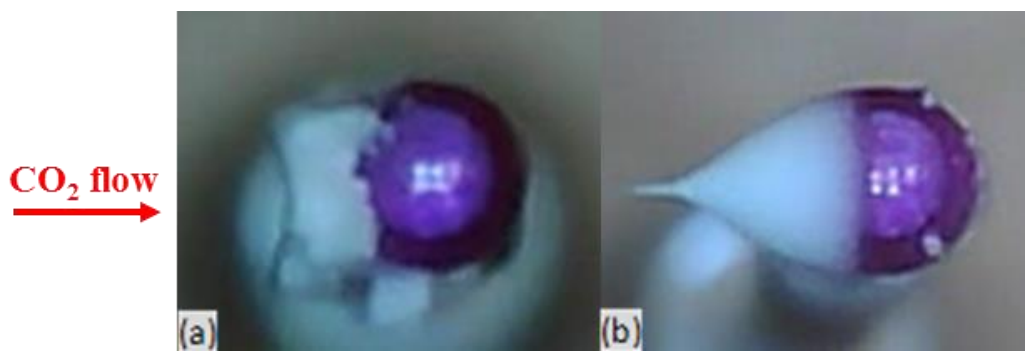

Figure 16 Build-up of snow particles on the stylus due to prolonged exposure to snow stream, in the form of (a) snow and (b) ice

Snow build-up tended to occur when the stream was dominated by turbulent flow (i.e. long standoff distance), while ice build-up tended to occur when the stream was dominated by laminar flow (i.e. short standoff distance). In either case, build-up of snow particles effectively shielded the tip surface from the stream, thus impeding further cleaning. Furthermore, a sharp increase in the drag force was observed as snow particles gradually built up on the stylus, as shown in Figure 17.

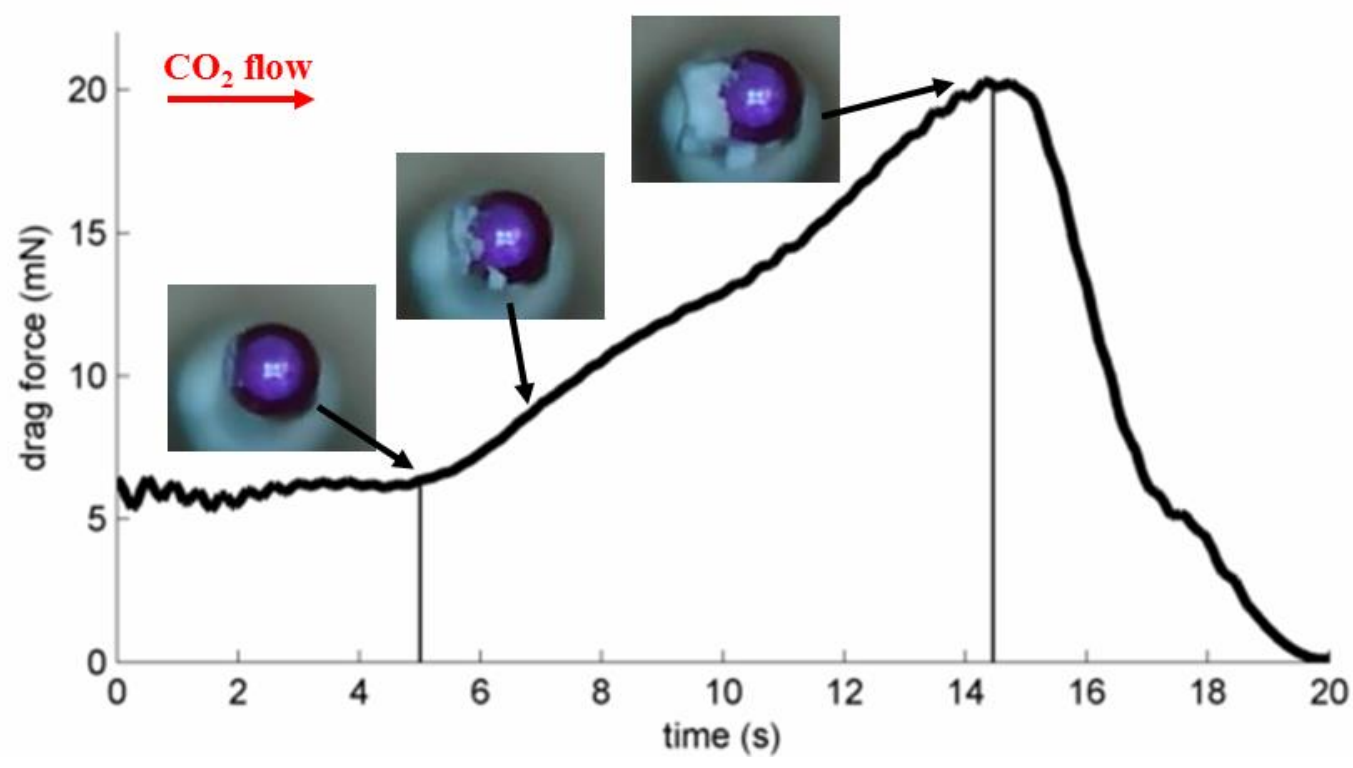

Figure 17 Increase in imparted drag force during build-up of snow particles

Build-up of snow particles did not dissipate over time until the snow stream was switched off, when the drag force had become three times higher than expected. Although thermal impact due to exposure to the snow stream did not cause direct damage to the stylus, it increased the risk of mechanical damage to the stylus shaft. In addition, severe condensation on the stylus tip was found. As a result, the stylus needed to dry up in the air and return to room temperature before it can be used for probing.

The coaxial setup of the nozzle used in the assisted unit produced a stream that diverged into a wider diameter over distance. As a result, the stylus shaft and mount also became exposed to the snow stream, 
not only causing the drag force to increase but also risking damaging sensitive electronic components of the probe. In comparison, the handgun unit using only $\mathrm{CO}_{2}$ is a simpler system with flexible components and a snow stream that does not diverge over the working distance, as shown in Figure 10. For these reasons, the pure $\mathrm{CO}_{2}$ snow cleaning approach made for a good candidate for precision cleaning of $\mu \mathrm{CMM}$ styli.

Thermal impact on the stylus due to exposure to $\mathrm{CO}_{2}$ may affect measurement accuracy due to distortion in the stylus geometry from thermal expansion. In this case, the stylus cannot be used for probing until it has returned to room temperature; additional air flow may be required to shorten this period.

\section{Prototype device for in situ stylus cleaning}

$\mu \mathrm{CMM}$ styli are often complex, costly and prone to damage during handling. In addition to the coverage and imparted drag force issues discussed above, other restraints apply to in situ cleaning such as small workspace, complicated stylus assembly and sensitivity to temperature. For example, the stylus used in the Zeiss F25 $\mu \mathrm{CMM}$ consists of a ruby sphere of $125 \mu \mathrm{m}$ diameter on a shaft made of tungsten carbide mounted to a silicon sensor. As the ruby sphere is the component that makes contact it must be kept clean, while exposing the shaft or the silicon chip to the snow stream is likely to cause overloading and damage. Therefore, the cleaning process needs to be not only gentle but also targeted, with limited thermal impact and full coverage of the stylus tip.

To address these challenges, specific features were incorporated into the pure $\mathrm{CO}_{2}$ snow cleaning approach, and a prototype device has been developed, as shown in Figure 18.

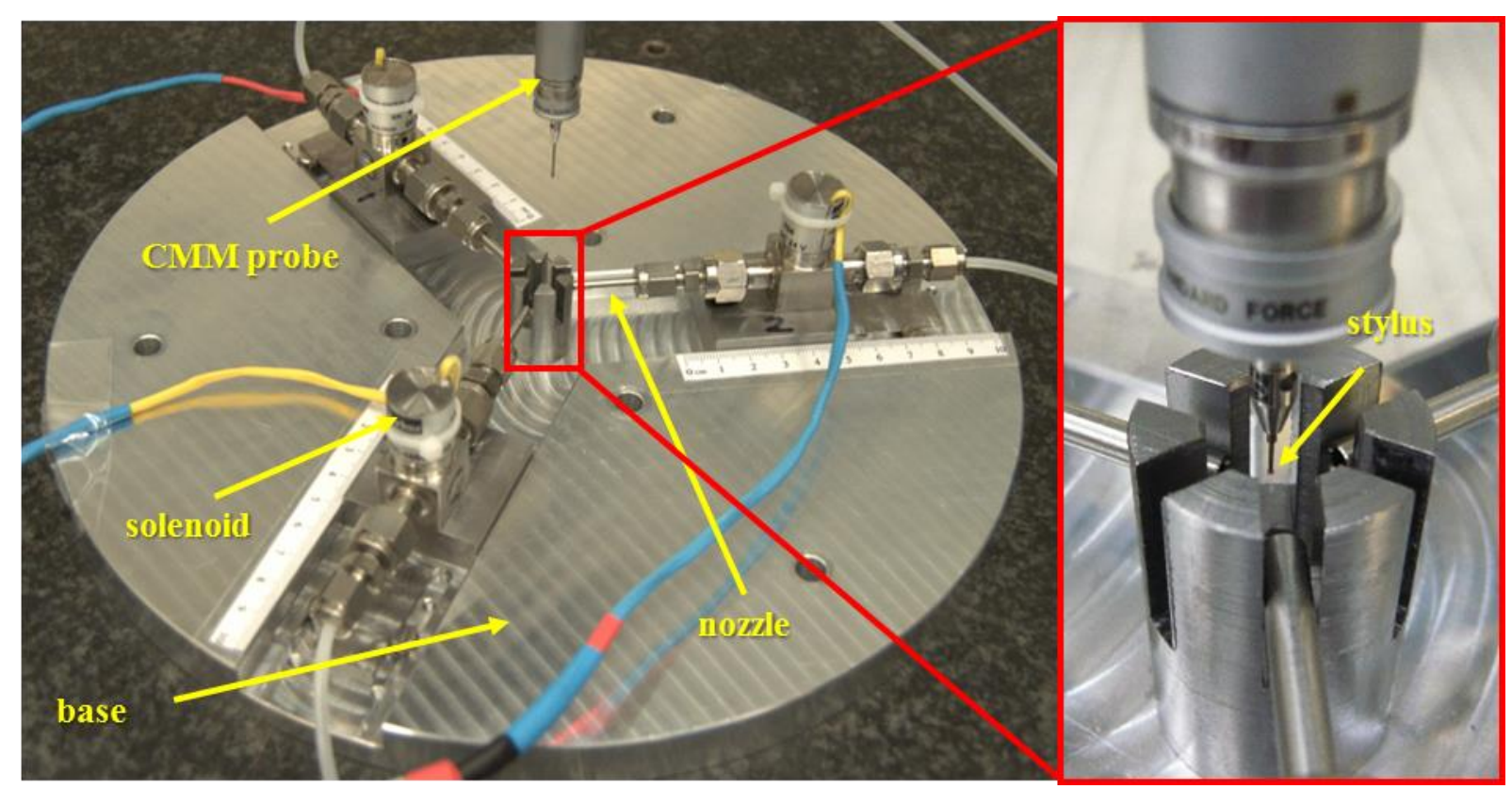

Figure 18 A prototype device for in situ cleaning of $\mu \mathrm{CMM}$ stylus tips using $\mathrm{CO}_{2}$ snow [11]

The developed prototype device included several useful features: 
(1) Nozzle with a small orifice and long exit, which produces sub-millinewton to millinewton level imparted drag force for safe cleaning. It also creates a non-divergent snow stream with about 2 $\mathrm{mm}$ diameter to ensure only the tip of the stylus is exposed to the snow stream.

(2) Solenoid switching of snow stream, which allows for multiple short pulses (less than $1 \mathrm{~s}$ duration) of snow stream to be used instead of a continuous stream. This prevents prolonged exposure to the snow stream and alleviates condensation. Pulse width is programmable using a microcontroller such as an Arduino Uno.

(3) Three identical nozzles being placed symmetrically around the stylus, which allows the entire stylus tip to be cleaned in a single procedure without the need for stylus rotation. Furthermore, such symmetrical setup enables the three snow streams to balance each other out and in theory generate zero net imparted drag force.

The focused snow stream produced minimises unwanted effect on the other parts of the stylus or probe. Additionally, by exposing only the tip sphere to the stream, imparted drag force is reduced without compromising cleaning effects. The internal geometry of the nozzle imaged using X-ray computed tomography is shown in Figure 19.

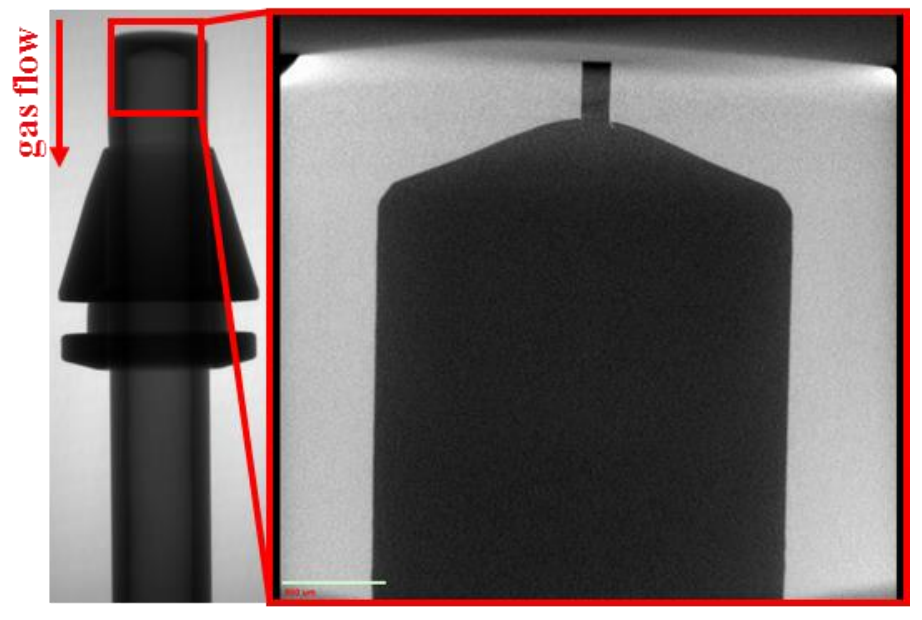

Figure 19 Cross section of the nozzle used in the developed prototype device

The nozzle used an orifice of $140 \mu \mathrm{m}$ to limit the flow rate and affect imparted force. A rapid expansion zone allowed the snow particles to form in the stream that was then guided through a long tube of $2 \mathrm{~mm}$ diameter and aspect ratio over 10. Imparted drag force can be adjusted by changing the diameter of the orifice. While a focused narrower stream can be produced by reducing the diameter of the inner tube, sufficient expansion zone must be allowed to produce solid $\mathrm{CO}_{2}$ particles.

The solenoid valves require electrical circuits to operate, which increased the complexity of the device. However, it enabled the important automated cleaning procedure working collaboratively with the $\mu \mathrm{CMM}$ system without human intervention. 
Although a single snow stream cannot reach the occluded side of the stylus tip, the use of three streams was sufficient to cover the entire sphere, as shown in Figure 20; the adhesive below the sphere in Figure 20(b) appeared white but was not part of contamination. This feature complies with the fact that most $\mu \mathrm{CMMs}$ do not have a rotational axis.

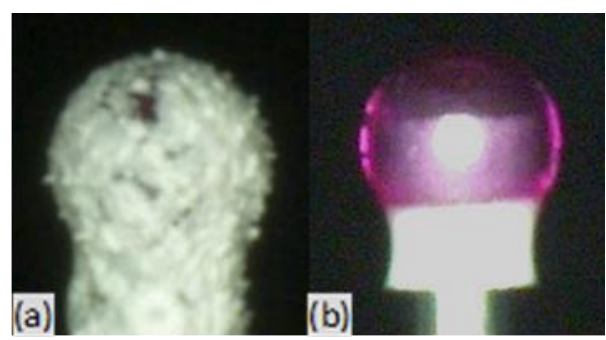

Figure 20 A contaminated stylus tip (a) before and (b) after snow cleaning with the developed prototype device

Zero net drag force was not achieved, due to fluctuation in the streams and imperfect alignment of the nozzles. However, at $1 \mathrm{~cm}$ standoff distance, net imparted force has been successfully reduced to 0.11 $\mathrm{mN}$. For better balance of imparted force, fluctuation in the snow stream, possibly caused by the frosting on the nozzle, needs to be addressed. One potential solution is the use of assisted heating or simply compressed air, which can also help remove condensation and return the stylus to room temperature in a shorter period.

The benefit of reduced net imparted force using symmetrically oriented streams also creates the opportunity of using stronger streams to remove well adhered debris while keeping the imparted force low, which is worth further investigation.

For the purpose of further characterisation studies, the diameter of the presented prototype is $300 \mathrm{~mm}$ allowing movement of the nozzles. However, a more compact device within $80 \mathrm{~mm}$ in diameter can be achieved with modified design. Its size can be further reduced if the nozzles are machined into a single part with integrated solenoid valves.

\section{Conclusions}

This study has experimentally explored the suitability of removing surface debris on $\mu \mathrm{CMM}$ stylus tips using $\mathrm{CO}_{2}$ snow. Two types of snow cleaning devices were investigated: a pure $\mathrm{CO}_{2}$ handgun unit and a two-gas system with accelerated flow. The potential damage to the stylus and cleaning efficacy of snow cleaning has been evaluated. Cleaning at millinewton force level was found to be effective except for well adhered debris. No sign of abrasion by snow particles was observed on the stylus tip surface. Taking into account the challenges put forth by in situ cleaning, the pure $\mathrm{CO}_{2}$ snow cleaning approach was preferred. Subsequently, a prototype snow cleaning device has been developed, incorporating several features to address the challenges of in situ stylus cleaning. The developed prototype has successfully achieved: 
- effective and targeted stylus cleaning,

- safe cleaning at low imparted force,

- full coverage of the stylus tip without the need for stylus rotation, and

- in situ and automated cleaning capability.

With these combined characteristics, this automated in situ cleaning device enables efficient and regular cleaning of $\mu \mathrm{CMM}$ and allows improvement of measurement accuracy without punishing measurement throughput.

\section{Acknowledgements}

This study was funded by the European Metrology Research Programme (EMRP). The EMRP is jointly funded by the EMRP participating countries within EURAMET and the European Union. The authors would like to appreciate the support of the Precision Manufacturing Centre at the University of Nottingham for assisting this project.

\section{References}

[1] Thalmann R, Meli F, Küng A. State of the Art of Tactile Micro Coordinate Metrology. Applied Sciences. 2016; 6(5): 150

[2] Sheu D-Y. Manufacturing tactile spherical stylus tips by combination process of micro electro chemical and one-pulse electro discharge technology. Int J Adv Manuf Technol. 2014; 74(58): 741-747

[3] Chao ZX, Tan SL, Xu G. Evaluation on the probing error of a micro-coordinate measuring machine. in SPIE 7155. 2008.

[4] Flack D, Claverley J, Leach R. Chapter 9 - Coordinate Metrology. In: R Leachs, editor. Fundamental Principles of Engineering Nanometrology (2nd Edition), Oxford: William Andrew Publishing; 2014

[5] Anaïs N, Alain K, Felix M. Study of sapphire probe tip wear when scanning on different materials. Meas Sci Technol. 2012; 23(9): 094016

[6] Brousseau E, Al-Musawi RSJ, Lebiez D. A hybrid roll-to-roll AFM set-up for high throughput tip-based nano-machining. Manufacturing Letters. 2015; 6: 10-13

[7] Gaoliang D, Sebastian B, Frank P, Hans-Ulrich D. A high precision micro/nano CMM using piezoresistive tactile probes. Meas Sci Technol. 2009; 20(8): 084001

[8] International Organisation for Standardization. ISO 10360-5:2010 Geometrical product specifications (GPS) - Acceptance and reverification tests for coordinate measuring machines (CMM) - Part 5: CMMs using single and multiple stylus contacting probing systems. 2010: Geneva, $\mathrm{CH}$

[9] Kinnell PK, Habeb RR. An evaluation of cleaning methods for micro-CMM probes. Meas Sci Technol. 2013; 24(8): 085603

[10] Feng X, Lawes S, Kinnell P. Evaluation of the Capabilities and Damage Risk of Cleaning Methods for Micro-CMM Stylus Tips, in Proceedings of the 4M/ICOMM2015 Conference. 2015: Milan, Italy

[11] Feng X, Lawes S, Kinnell P. The development of a snow cleaning system for micro-CMM stylus tips. in Proc. 15th Int. euspen Conf. 2015. Leuven, Belgiam 